\title{
DESIGN OF A MOBILE ROBOT TO WORK IN HOSPITALS AND TRAJECTORY PLANNING USING PROPOSED NEURAL NETWORKS PREDICTORS
}

\author{
Şahin Yıldırım ${ }^{1}$, Sertaç Savaș ${ }^{1}$ \\ ${ }^{1}$ Mechatronics Engineering Department, Engineering Faculty, Erciyes University, Kayseri 38039, Turkey \\ Emails: sahiny@erciyes.edu.tr,sertacsavas@erciyes.edu.tr
}

\begin{abstract}
Considering the intense and tiring working conditions in hospitals, healthcare personnel's performance decreases during prolonged working times, and patients are directly affected by this decrease in performance. This study aims to design and implement a mobile robot that can help healthcare professionals improve the healthcare industry conditions. In this context, the focus is on the mobile robot performing two main tasks. The first task is dispensing medication to patients with an eight-chamber mechanical feeding unit. Thus, patients can take only their medicines from the defined reservoir by selecting their names or photos on the touch screen. The second task is to interact with patients to give moral support with phrases such as "good morning", "you look great today". Also, drug delivery activity is recorded in a database, and the health status of the patients can be kept under surveillance with the camera on the mobile robot. The designed mobile robot goes to the patient rooms with magnetic strip tracking. For this purpose, a controller is designed for the omni-drive robot using MATLAB, and its performance is simulated. Also, the control velocities that enable tracking the trajectories are taught to artificial neural networks (ANN), and the requirement magnetic strip for trajectory tracking is eliminated. In this direction, two artificial neural networks are compared in terms of their learning performance.
\end{abstract}

Keywords: Mobile hospital robot, Omni-drive, Controller design, Trajectory tracking, Neural networks.

\section{Introduction}

Developing technology has brought robotic systems from factories to our daily lives. Mobile robots, which find many application areas, especially with their mobility features, are among the most important research topics of recent years. Until today, many types of mobile robots have been used to solve different problems. Also, robots used in mobile applications have very different motion systems [1], and the movement patterns of animals generally inspire them in their designs. Although the existence of a wheel or similar system in the animal kingdom is not complete, the wheel design is inspired by the rolling motion of some insects [2].

Omni-directional wheels are used in many studies on mobile robots due to their driving flexibility. Kosuge et al. [3] developed a mobile robot called Ms. Dancer. This mobile robot was designed for dance partnership, using omni-directional wheels in the motion system. Human-robot physical interaction and coordination were investigated based on ball dances with the developed robot. This study is an excellent example of mobile robot applications' breadth and the flexibility of omnidirectional capability. Many studies have also been done on the control and trajectory tracking of omnidirectional mobile robots. Wang et al. [4] used a model predictive control strategy to control an omnidirectional mobile robot's trajectory tracking. Liu et al. [5] developed a non-linear controller consisting of a two-stage structure. While the kinematic control approach is applied in the controller's outer loop, the Trajectory Linearization Control (TLC) method is used in the inner loop, which is based on the robot's dynamic model. Karras and Fourlas [6] designed a fault-tolerant control scheme to control an omnidirectional mobile robot and accordingly developed a non-linear model predictive controller.

Studies have also been carried out for different configurations of omni-directional mobile robots. Wang et al. [7] performed trajectory tracking control with the robust model predictive control (MPC) strategy for a four-mecanum-wheeled omnidirectional mobile robot (FM-OMR). Cooney et al. [8] also designed a four-mecanum-wheeled omnidirectional mobile robot and performed path tracking. They used an optical mouse to navigate the mobile robot with a dead-reckoning approach. Hashemi et al. [9] also designed a controller for a four-wheeled omni-directional mobile robot. In their work, they designed a model-based PI-fuzzy 
controller. They used the mobile robot's linear discrete dynamic model to determine the optimum inputs. Mishra et al. [10] used the behavioral faulttolerant-control approach for a four-mecanumwheeled omni-directional mobile robot.

Mobile robots are frequently used in the healthcare field. Tan et al. [11] developed a robotic system for transporting surgical tools in hospitals. They proposed a robust integrated system for enabling mobile robots to autonomously perform manipulation of assets. Wang et al. [12] designed a smart robotic hospital bed to transport critical patients in crowded hospital corridors safely. Again, due to their driving ability, omni-directional wheels are frequently preferred in mobile robot designs to be used in a hospital environment. Takahashi et al. [13] designed an omni-directional mobile robot to be used in transport applications in the hospital domain. They used a four-wheel configuration in the driving system of the robot they designed. Saekow et al. [14] designed an autonomous rehabilitation robot and implemented external force/velocity control.

This paper is organized into six sections. In section 2, mechanical, electronic, and software designs of the mobile robot are explained. In section 3 , kinematics of the mobile robot is given. In section 4 , controller design for trajectory tracking, and in section 5, ANN training of control velocities that will track the determined trajectories is mentioned, study results and findings are given. In the last section, the 6th section, the results, and suggestions are emphasized.

\section{Mobile Robot Design}

The mobile robot will dispense medicine to patients with an eight-chamber mechanical feeding unit controlled through the interface of a touch screen. Thus, patients will be enabled to take only their medication from the defined reservoir by selecting their names or photos on the screen. Also, the mobile robot will interact with the patients with words such as "good morning", "you look great today" to give moral support to the patients. In addition to these basic tasks, drug delivery activity will be recorded in a database, and the general health status of the patients will be recorded with a camera on the mobile robot to ensure that the healthcare personnel keep the patients under surveillance. In this direction, the design process of the study consists of three main parts: mechanic, electronic, and software.

The mechanical parts of the mobile robot consist of;

- Drive system that enables navigation

- A mechanism for drug delivery

- The general construction of the robot
The mobile robot is driven by three omnidirectional wheels to be placed at $120^{\circ}$ angles and three different servo motors for each wheel. These wheels provide the robot omni-drive. Also, these wheels are specially designed and manufactured to increase the ground grip and increase the carrying capacity of the mobile robot. Omni-directional wheels and servo motors manufactured to be used in the mobile robot's driving system are shown in Figure 1.

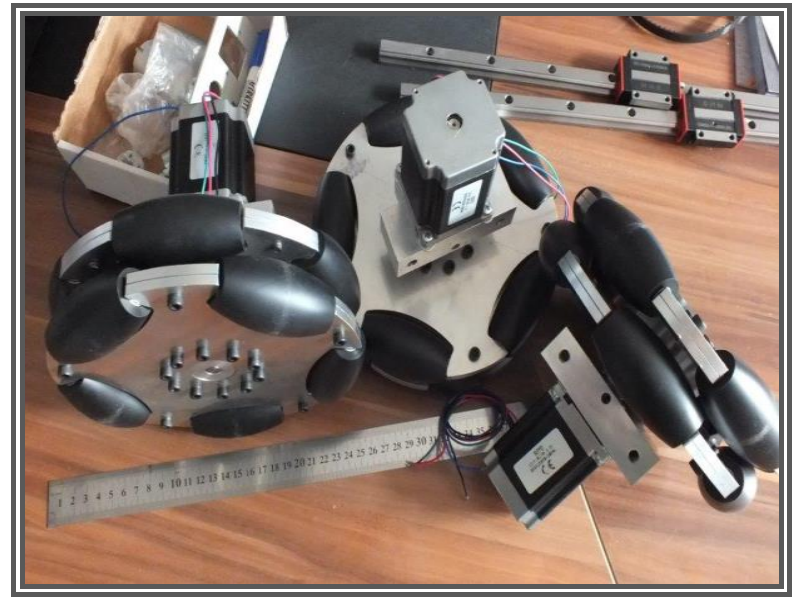

Figure 1: Omni-directional wheels and servo motors used in mobile robot driving system.

Also, a rotating mechanism with eight chambers that can deliver drugs to eight different patients is designed. After the patient selection is made on the robot screen, the relevant chamber is positioned in front, and its cover is opened. The CAD (computer-aided design) model of the drug delivery mechanism is given in Figure 2. Figure 3 shows the drug delivery slot on the prototype mobile robot. The outer shell of the mobile robot is made of fiberglass. Figure 3 also shows the CAD model of the mobile robot.

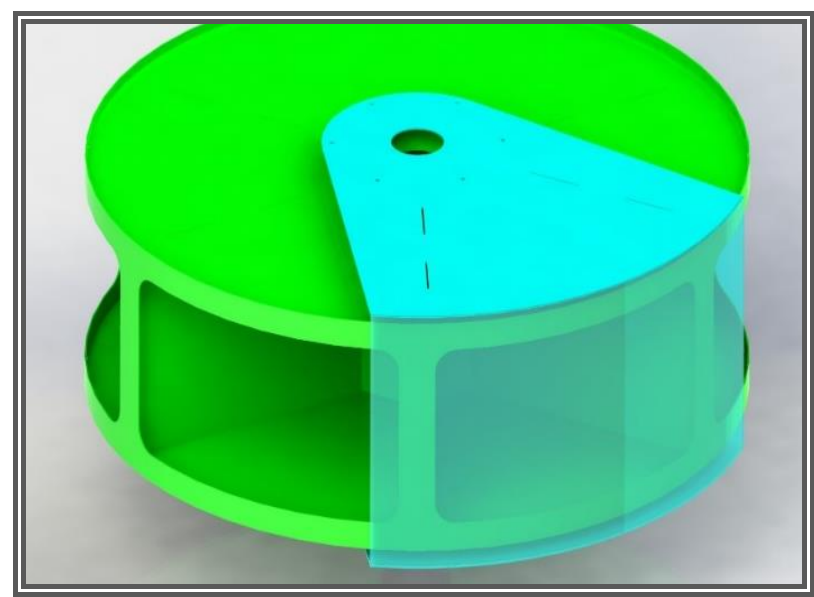

Figure 2: Mobile robot drug delivery mechanism CAD model 


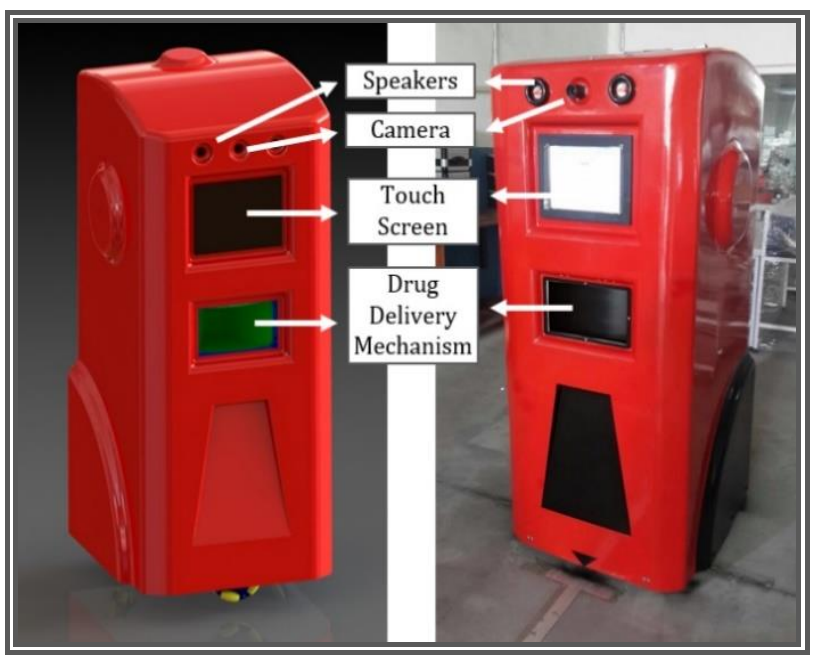

Figure 3: CAD model of the mobile robot and the produced prototype model

Mobile robot electronic equipment: It consists of a control card, communication hardware, sensors placed under the mobile robot to detect magnetic lines, motor drivers, two batteries, an LCD touch screen, two speakers for audio notifications, a camera placed between the speakers and a charging station where it will be charged in standby mode.

The third stage of the design is developing the software; navigation software to go to patient rooms, software to provide medication distribution, database software to keep records for transactions, and communication software to communicate with a computer when necessary.

\section{Kinematics of Three-Wheel Omnidirectional Drive}

Three omni-directional wheel configuration used in the mobile robot drive system is given in Figure 4. In order to make inverse kinematic calculations [1] in global coordinates, the linear $\left(v=\sqrt{x^{2}+y^{2}}\right)$ and angular velocity $(\varphi)$ of the mobile robot must be defined. The velocity of each wheel consists of tangential and radial components. First wheel velocity components and total velocity are given in Equation 1-4. $\dot{x}^{x}$ and $\dot{y}$ are the components of the linear velocity in the $\mathrm{x}$ and $\mathrm{y}$ axes, and $\mathrm{R}$ is the distance between the center of the mobile robot and the wheels.

$$
\begin{gathered}
v_{1}=v_{1 t}+v_{1 r} \\
v_{1 t}=-\dot{x} \sin (\varphi)+\dot{y} \cos (\varphi) \\
v_{1 r}=R \dot{\varphi} \\
v_{1}=-\dot{x} \sin (\varphi)+\dot{y} \cos (\varphi)+R \dot{\varphi}
\end{gathered}
$$

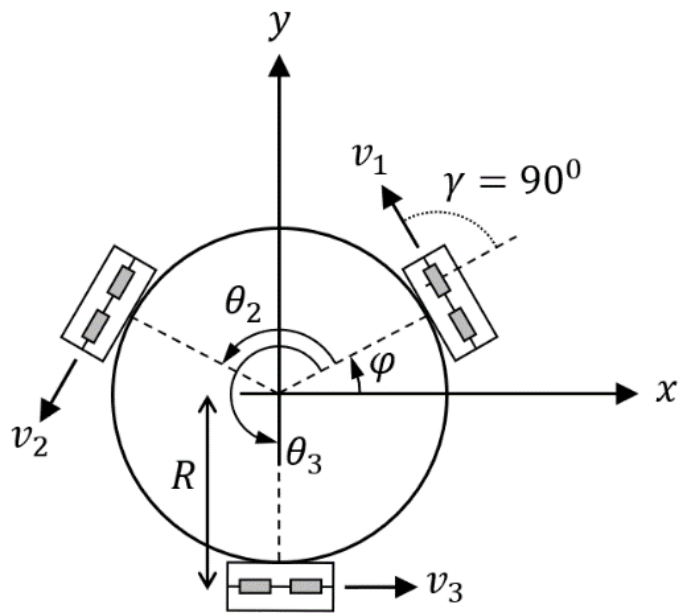

Figure 4: Schematic model of three-wheel omnidirectional drive

Since the wheels are placed at 120 degrees, $\theta_{2}$ is 120 degrees and $\theta_{3}$ is 240 degrees. Similarly, the second and third wheel velocities are given in Equation 5-6. Inverse kinematics $(v=J q)$ in global coordinates is expressed as in Equation 7.

$$
\begin{gathered}
v_{2}=-\dot{x} \sin \left(\varphi+\theta_{2}\right)+\dot{y} \cos \left(\varphi+\theta_{2}\right)+R \dot{\varphi} \\
v_{2}=-\dot{x} \sin \left(\varphi+\theta_{2}\right)+\dot{y} \cos \left(\varphi+\theta_{2}\right)+R \dot{\varphi} \\
{\left[\begin{array}{l}
v_{1} \\
v_{2} \\
v_{2}
\end{array}\right]=\left[\begin{array}{ccc}
-\sin (\varphi) & \cos (\varphi) & R \\
-\sin \left(\varphi+\theta_{2}\right) & \cos \left(\varphi+\theta_{2}\right) & R \\
-\sin \left(\varphi+\theta_{2}\right) & \cos \left(\varphi+\theta_{2}\right) & R
\end{array}\right]\left[\begin{array}{l}
\dot{x} \\
y \\
\dot{\varphi}
\end{array}\right]}
\end{gathered}
$$

For mobile robot control, the velocity components $\left(\mathscr{q}_{m}\right)$ in local coordinates must be defined. In this direction, forward kinematic equations $(\dot{q}=S v)$ are found by taking the inverse of the Jacobian matrix $(\dot{q}=S v)$. Finally, the mobile robot omni-drive forward kinematic equation [1] is given in Equation 8.

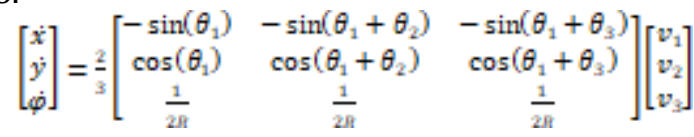

\section{Controller Design}

The mobile robot is modeled with MATLAB Simulink, and a controller is designed for line tracking. MathWorks Mobile Robotics Simulation Toolbox and Mobile Robotics Training Toolbox are used in the simulation studies. A PD controller is used as the controller, and the mathematical expression of the controller is given in Equation 9. Simulink block diagram of the mobile robot control loop is also shown in Figure 5.

$$
P D(s)=P+D \frac{N}{1+N-T_{x_{z}-1}}
$$




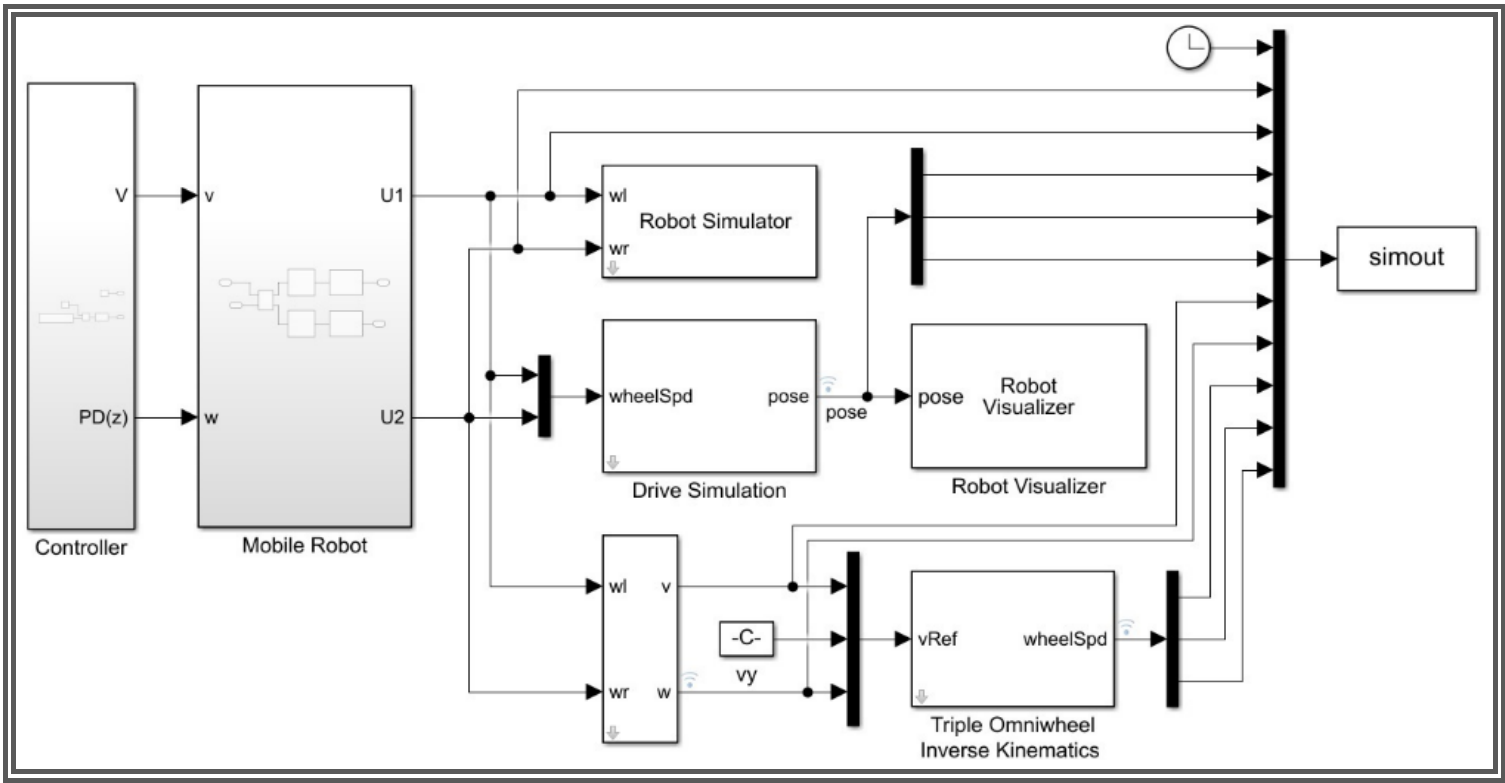

Figure 5: Simulink block diagram of the mobile robot control.

For the hospital scenario to be applied, the map in Figure 6 is designed, and the tracking performances are examined for two trajectories between "Starting Point - Patient Room 1" and
"Patient Room 1 - Patient Room 2". Mobile robot and controller parameters used in the study are given in Table 1.

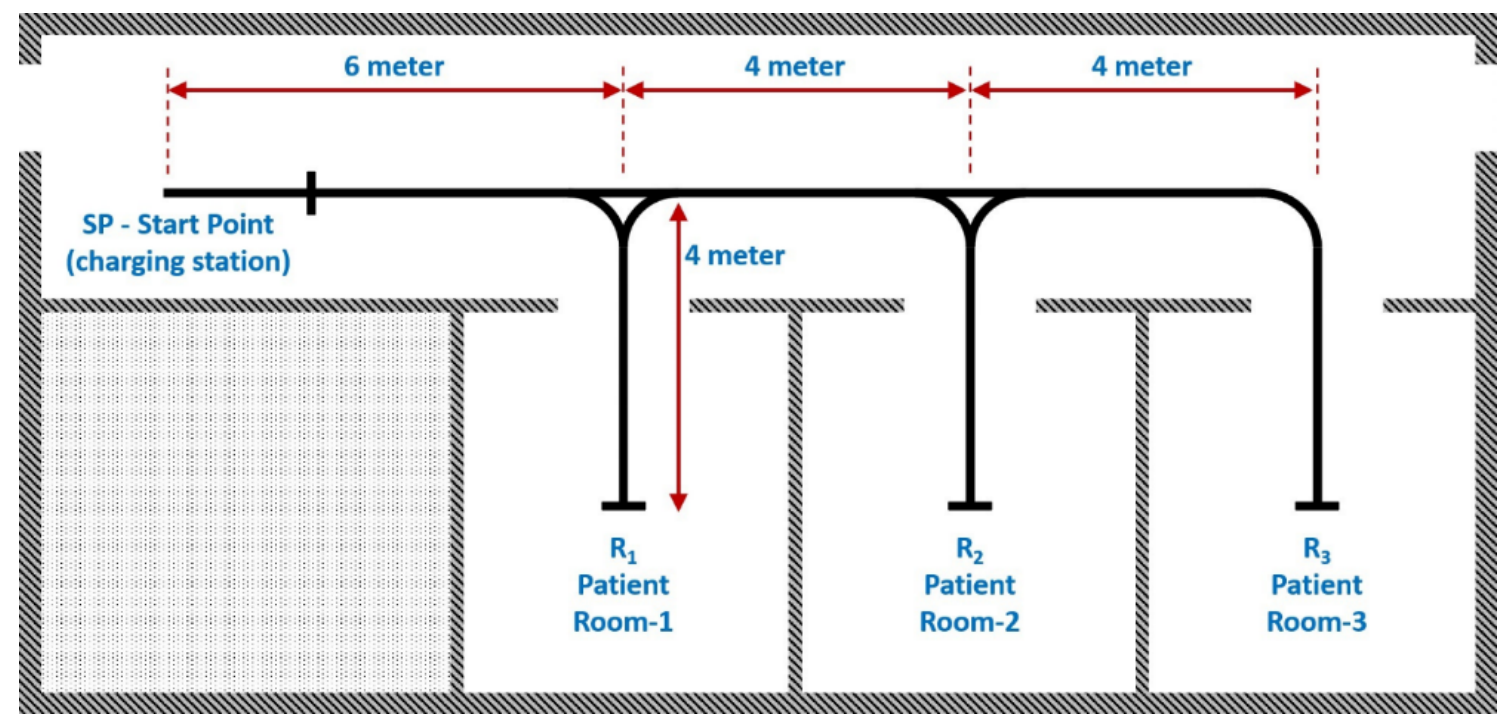

Figure 6: The map designed for the hospital scenario

Table 1. Mobil robot and control parameters

\begin{tabular}{llll}
\hline Parameter & Values & & \\
\hline Wheel Radius [m] & 0.125 & & \\
Robot Radius [m] & 0.4 & & \\
Wheel Angles [rd] & $\theta_{1}=0$ & $\theta_{2}=2.094$ & $\theta_{a}=-2.094$ \\
Sample Time = Ts [sec] & 0.01 & & \\
PD Controller Parameters & $P=0.01$ & $D=10^{-8}$ & \\
Filter Coefficient (N) & 100 & & \\
Start Point (SP) (charging station) & X Position $[\mathrm{m}]=1$ & Y Position $[\mathrm{m}]=5$ \\
Patient Room 1 (R1) & X Position $[\mathrm{m}]=7$ & Y Position $[\mathrm{m}]=1$ \\
Patient Room 2 (R2) & X Position $[\mathrm{m}]=11$ & Y Position $[\mathrm{m}]=1$ \\
\hline
\end{tabular}


The trajectory tracked by the PD controller between SP-R1 is given in Figure 7 , the reference heading angle and mobile robot heading angle on this trajectory are given in Figure 8, and the mobile robot posture error values are given in Figure 9. The trajectory tracked between R1-R2 is given in Figure 10 , the reference heading angle and mobile robot heading angle on this trajectory are given in Figure
11 , and the mobile robot posture error values are given in Figure 12. In addition to these studies, the mobile robot's scenario tests installed in Erciyes University, Mechatronics Engineering Department Laboratory are also performed. In this scenario, room navigation is carried out with magnetic strips placed on the floor and sensors that detect these magnetic strips.

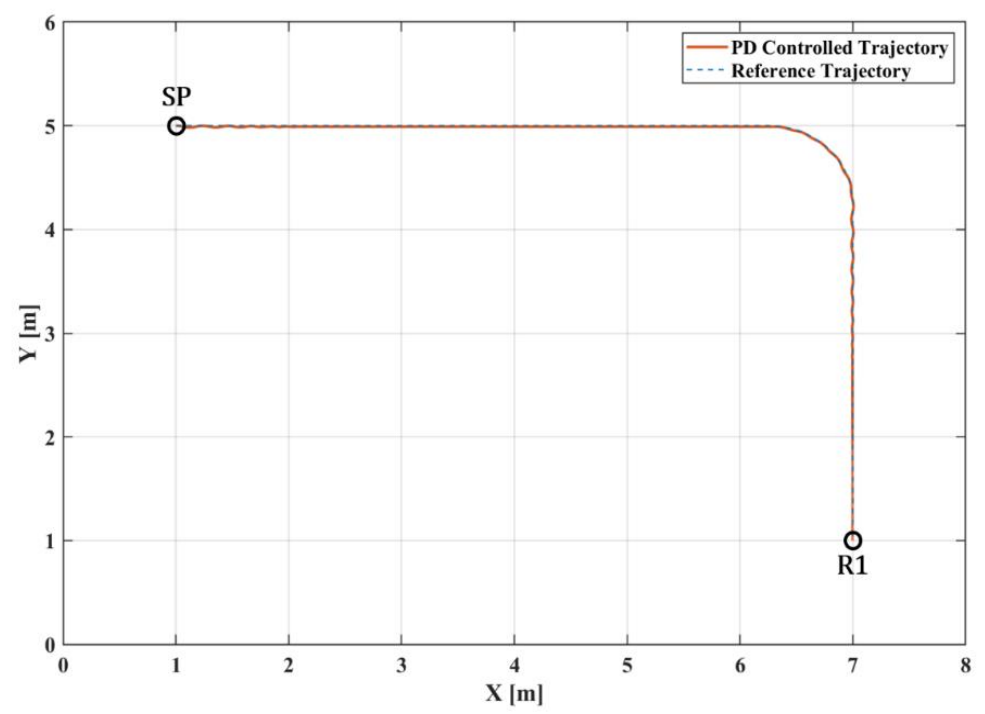

Figure 7: Reference and PD controlled trajectory between SP-R1

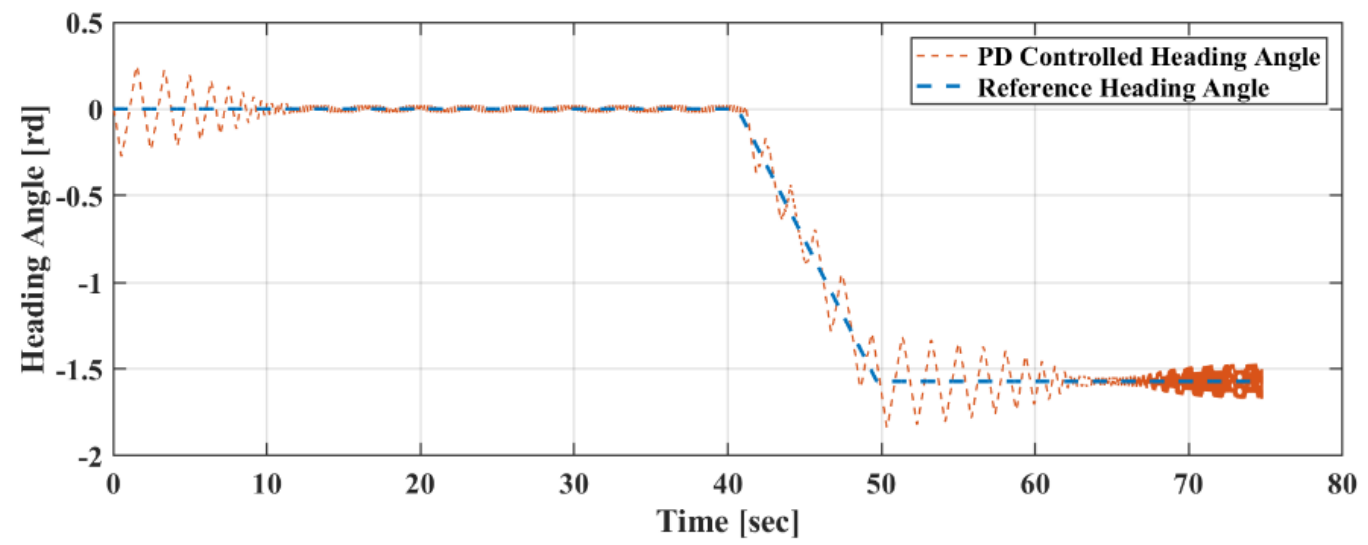

Figure 8: Reference and PD controlled heading angle between SP-R1

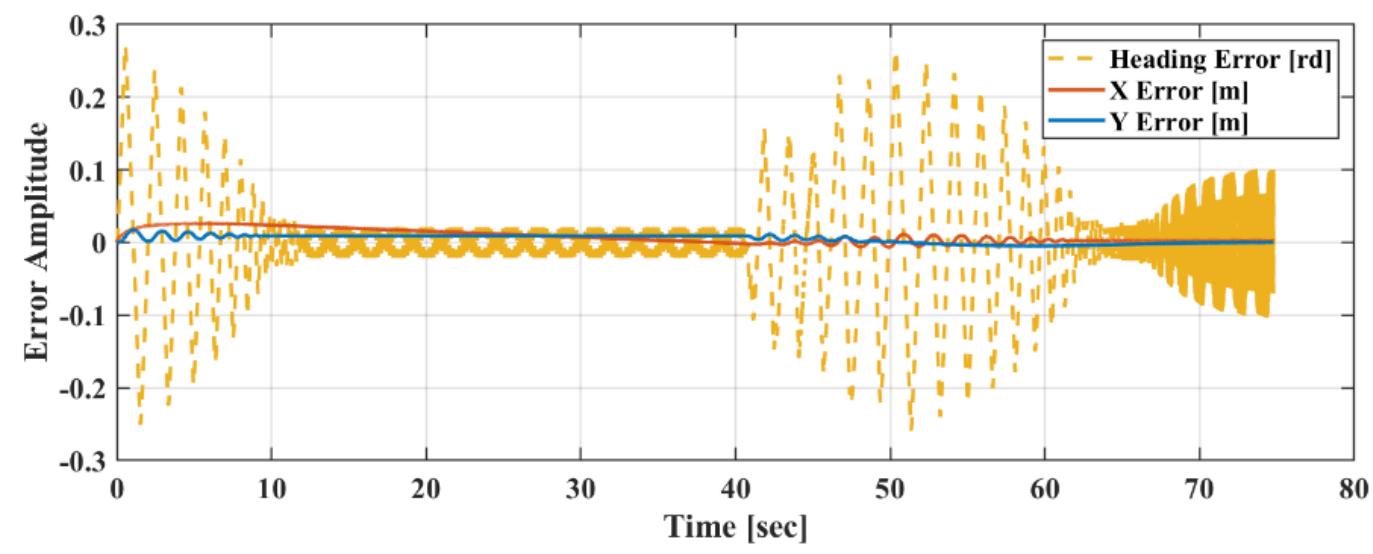

Figure 9: PD controlled mobile robot posture errors for trajectory between SP-R1 


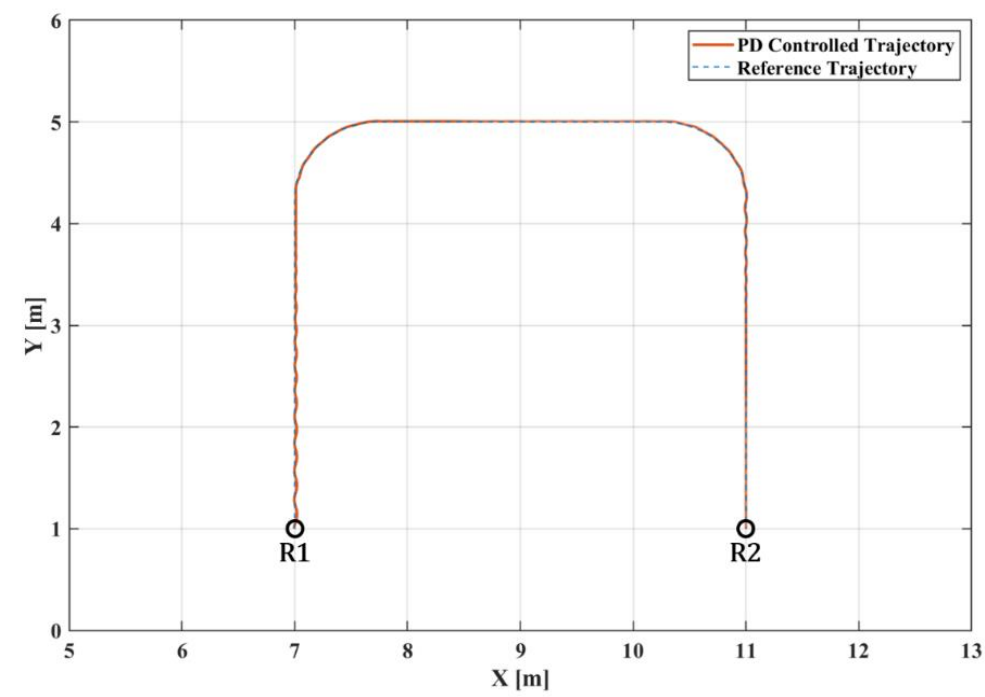

Figure 10: Reference and PD controlled trajectory between R1-R2

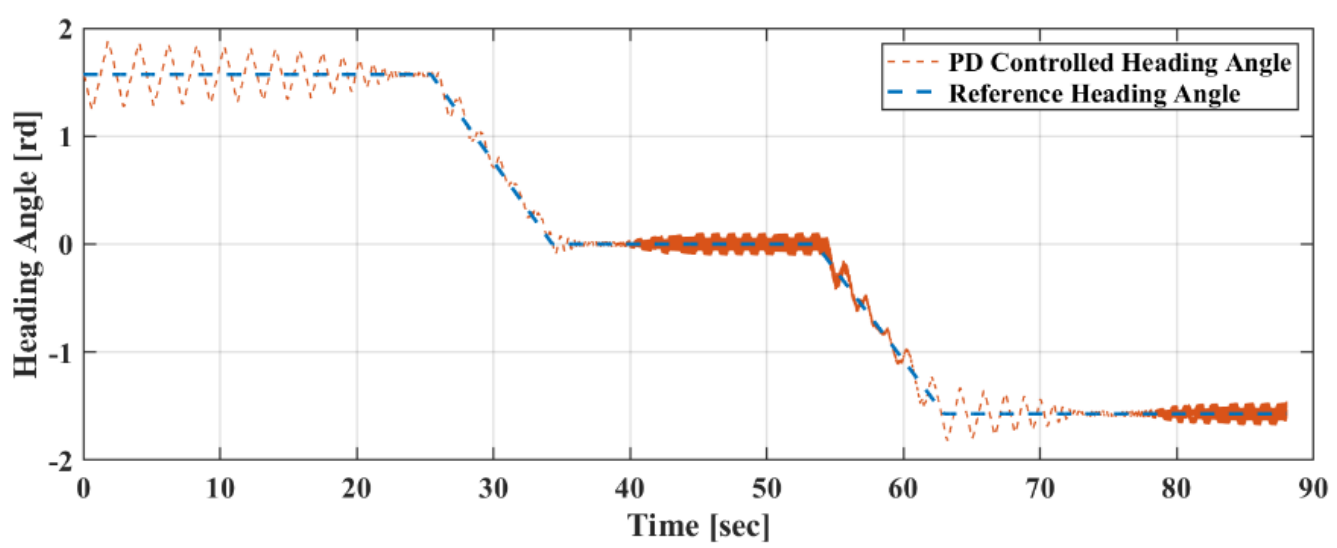

Figure 11: Reference and PD controlled heading angle between R1-R2

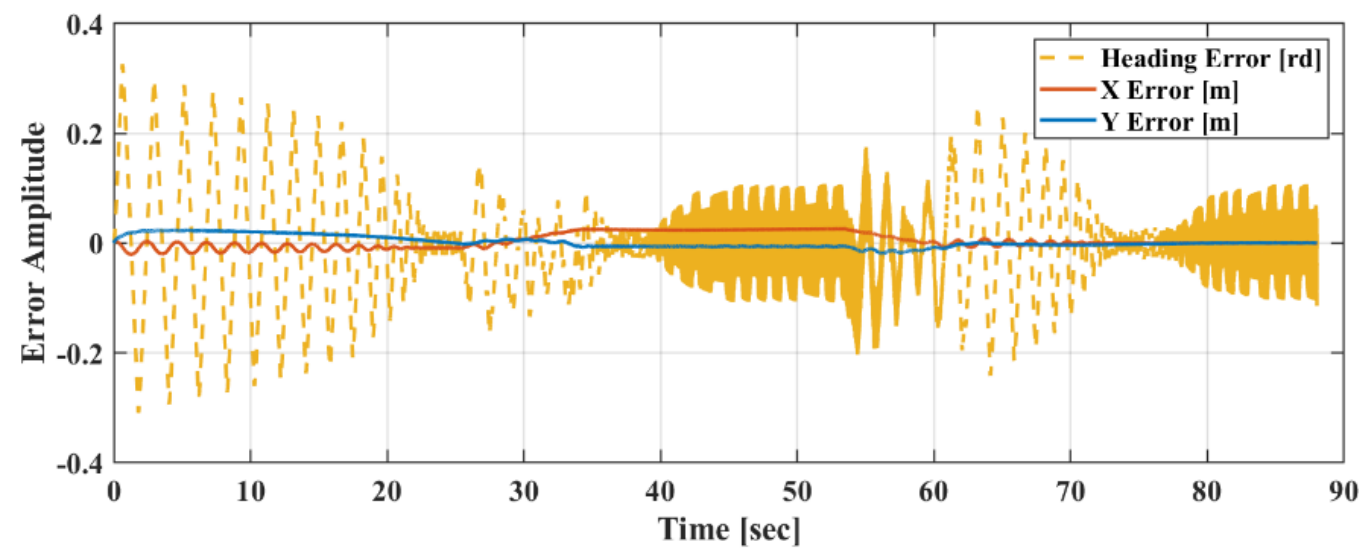

Figure 12: PD controlled mobile robot posture errors for trajectory between R1-R2

\section{Neural Network Training}

The system input is the linear and angular control velocity values " $v_{c}$ and $w_{c}$ " and the output of the designed control loop is the mobile robot posture " $\mathrm{x}$, $y$, and $\theta^{\prime \prime}$. The position values of two different trajectories tracked by the mobile robot and the control velocity values required to go to these positions are taught to the artificial neural networks.
In this way, the mobile robot's trajectories will be taught once with line tracking, and then the robot will be able to operate without the need for magnetic strips. Multilayer Perceptron (MLP) and Radial Basis Neural Network (RBNN) types are used for this purpose, frequently used in system modeling and fitting processes in artificial neural network training. Both network models are feed-forward neural networks with 3 inputs and 2 outputs and consist of a single hidden layer. 
The training process with artificial neural network and system inputs and outputs are given schematically in Figure 13.

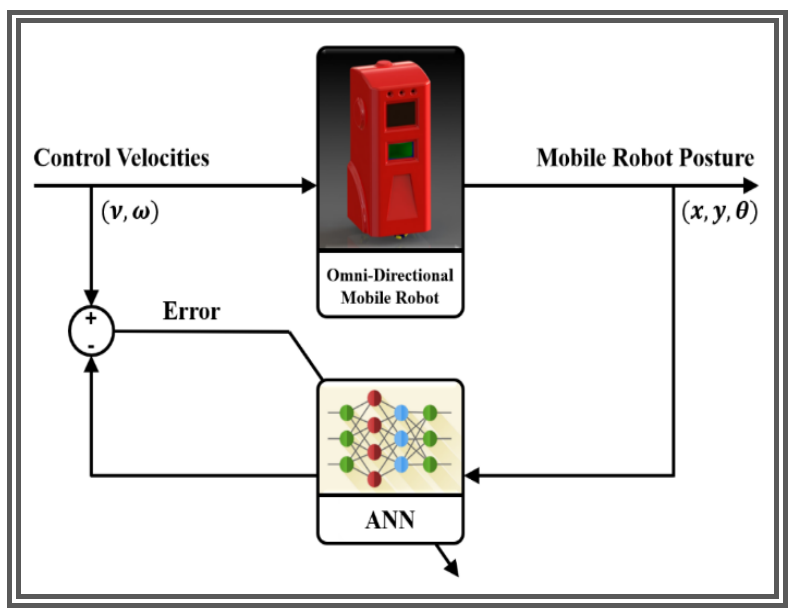

Figure 13: Training process block diagram with artificial neural network and system inputs and outputs
Training processes made for MLP are carried out for 3 different training algorithms (LevenbergMarquardt, Bayesian Regularization, Scaled Conjugate Gradient) and 3 different activation functions (logsig, purelin, and tansig). Also, the number of neurons $(\mathrm{H})$ in the hidden layer is changed between 1-100. Training parameters for MLP are given in Table 2. The lowest error value of each combination is given in Table 3 . Network and training parameters in the 6th row, which has the lowest MSE value, are selected.

Table 1. MLP training parameters

\begin{tabular}{lll}
\hline Parameter & Value \\
\hline Performance Function & MSE \\
Maximum Number of Iterations & 1000 \\
(Epochs) & & $1 \times 10^{-10}$ \\
Target Error Value & & 0.1 \\
Learning Rate (mu) & & \\
Number of Iterations to Show & 25 \\
Results & & \\
\hline
\end{tabular}

Table 2. MSE values for different MLP parameters

\begin{tabular}{|c|c|c|c|c|c|}
\hline \multirow[t]{2}{*}{ Training Algorithm } & \multirow[t]{2}{*}{ Activation Functions } & \multicolumn{3}{|c|}{$\begin{array}{c}\text { Number of } \\
\text { Neurons }\end{array}$} & \multirow[t]{2}{*}{ MSE } \\
\hline & & I & $\mathbf{H}$ & $\mathbf{0}$ & \\
\hline Levenberg-Marquardt & logsig & 3 & 59 & 2 & $3,0671 \times 10^{-3}$ \\
\hline Levenberg-Marquardt & purelin & 3 & 75 & 2 & $5,7089 \times 10^{-3}$ \\
\hline Levenberg-Marquardt & tansig & 3 & 36 & 2 & $0,1105 \times 10^{-3}$ \\
\hline Bayesian Regularization & logsig & 3 & 80 & 2 & $3,0698 \times 10^{-3}$ \\
\hline Bayesian Regularization & purelin & 3 & 30 & 2 & $5,71 \times 10^{-3}$ \\
\hline Bayesian Regularization & tansig & 3 & 62 & 2 & $0.0809 \times 10^{-3}$ \\
\hline Scaled Conjugate Gradient & logsig & 3 & 73 & 2 & $3,1519 \times 10^{-3}$ \\
\hline Scaled Conjugate Gradient & purelin & 3 & 46 & 2 & $5,7091 \times 10^{-3}$ \\
\hline Scaled Conjugate Gradient & tansig & 3 & 75 & 2 & $0,2358 \times 10^{-3}$ \\
\hline
\end{tabular}

Training operations for RBNN are performed by applying different spread values. The MSE values are given in Table 4 . The spread value in the 3rd row, which gives the lowest training error in the table, is selected for comparison.

Table 3. MSE values for different spreads of RBNN

\begin{tabular}{|c|c|}
\hline Spread & MSE \\
\hline 0.0001 & $1.9408 \times 10^{-31}$ \\
\hline 0.001 & $1.8743 \times 10^{-31}$ \\
\hline 0.01 & $0.4235 \times 10^{-31}$ \\
\hline 0.1 & $1.3658 \times 10^{-8}$ \\
\hline 1 & $1.2552 \times 10^{-4}$ \\
\hline 10 & $5.3398 \times 10^{-4}$ \\
\hline 100 & $2.1745 \times 10^{-3}$ \\
\hline
\end{tabular}

The control velocity outputs of MLP and RBNN networks trained with network parameters giving the lowest MSE values are compared with the actual velocity outputs. Actual linear control velocity graph for trajectory tracking between SP-R1 and linear control velocity graphs generated with MLP and RBNN are given in Figure 14, and graphs for angular control velocity are given in Figure 15. Likewise, the actual linear control velocity graph for trajectory tracking between R1-R2 and the linear control velocity graphs generated with MLP and RBNN are given in Figure 16, and the graphs for angular control velocity are given in Figure 17. As shown from the figures, RBNN has learned the actual control velocity values much more successfully. 


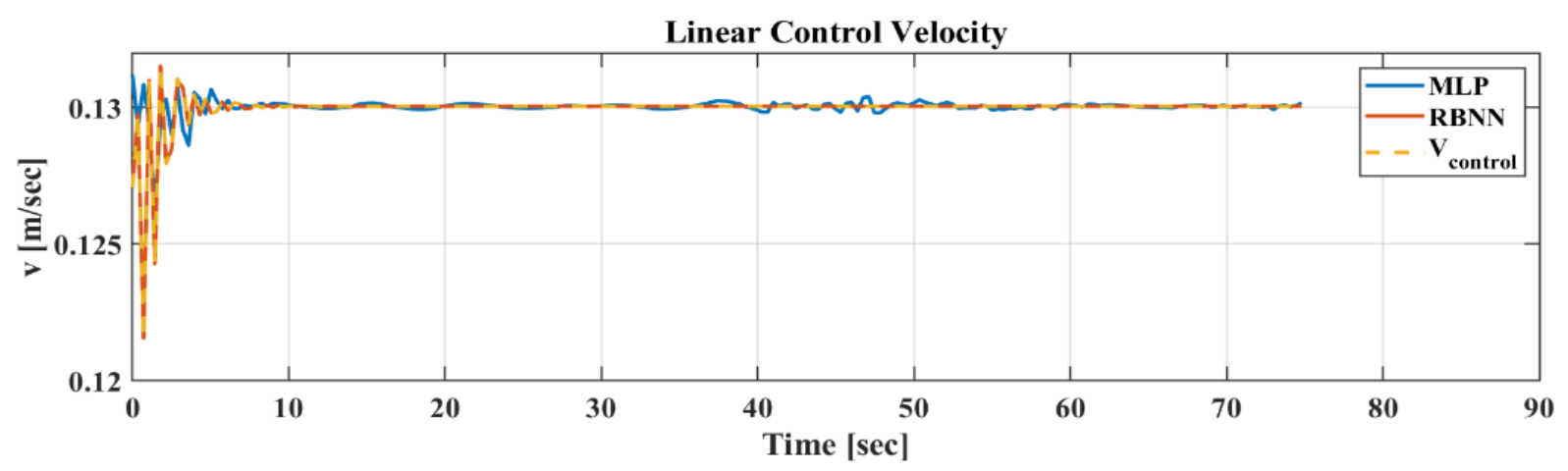

Figure 14: Linear control velocities generated with MLP and RBNN for trajectory tracking between SP-R1

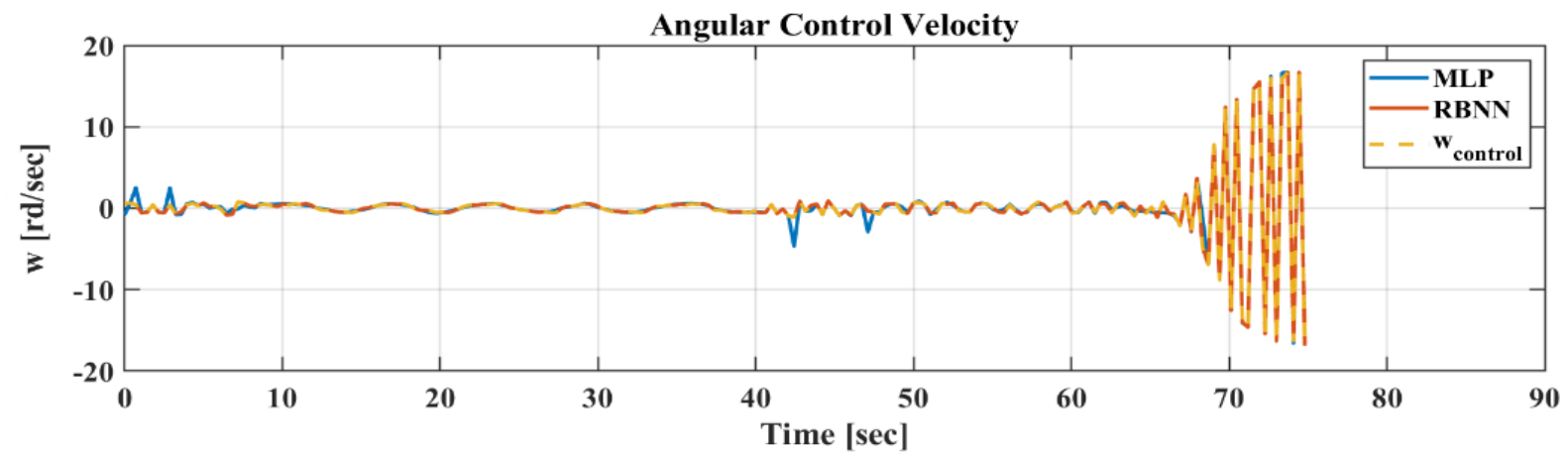

Figure 15: Angular control velocities generated with MLP and RBNN for trajectory tracking between SP-R1

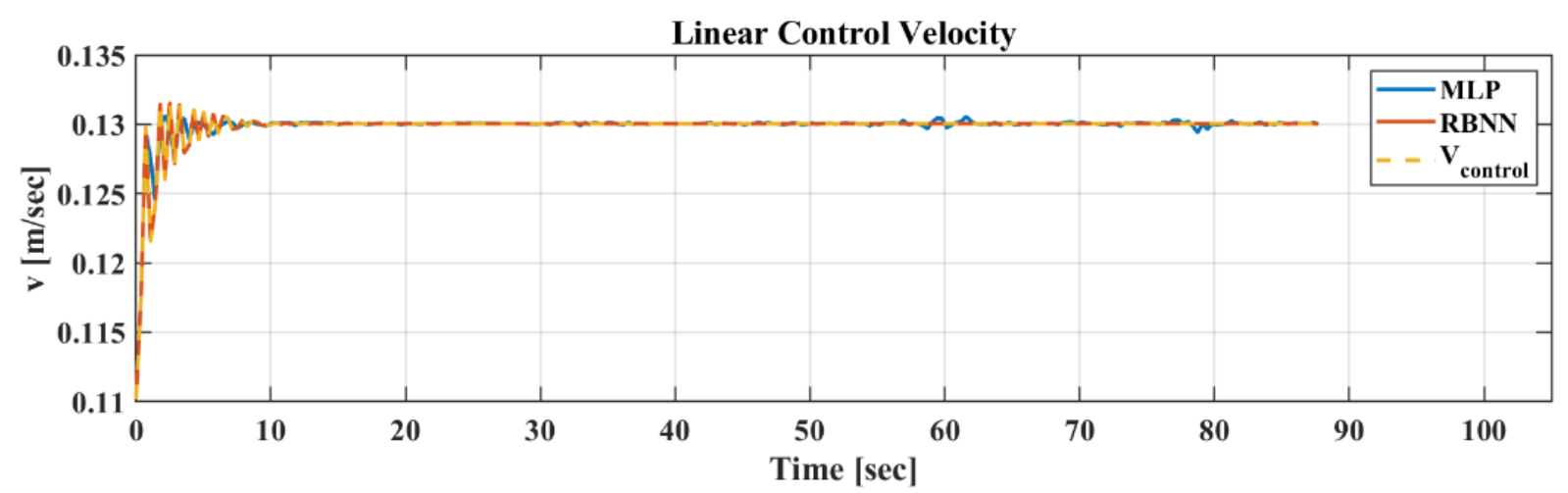

Figure 16: Linear control velocities generated with MLP and RBNN for trajectory tracking between R1-R2

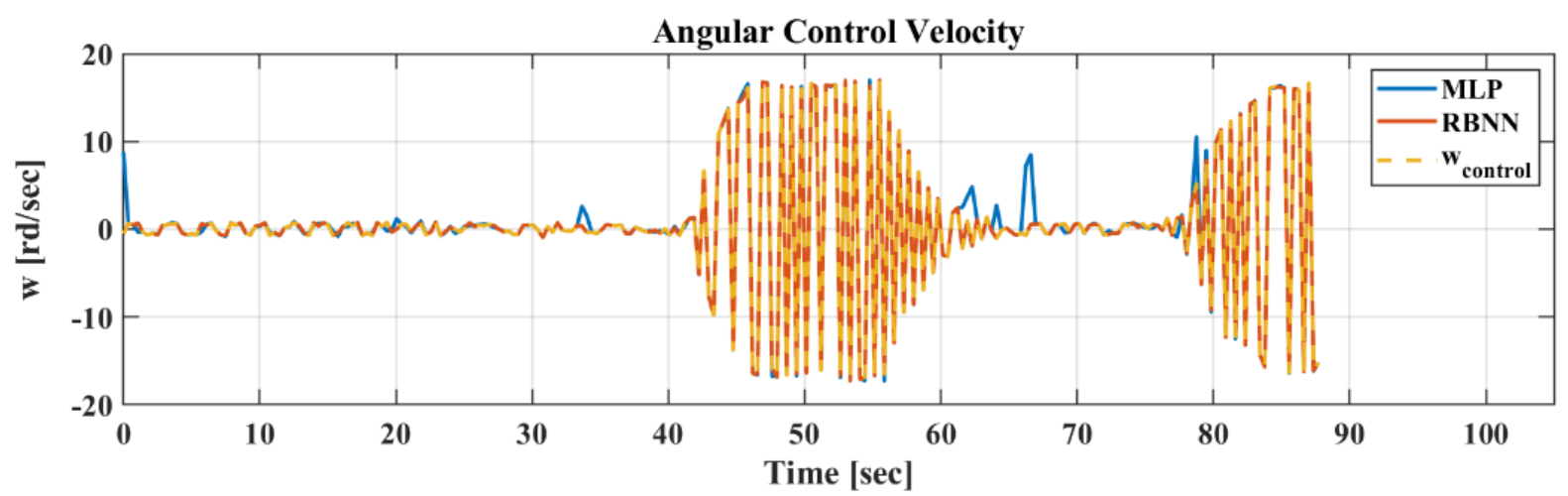

Figure 17: Angular control velocities generated with MLP and RBNN for trajectory tracking between R1-R2

\section{Conclusion and Future Research}

In this study, a mobile robot that can go to the patient room and automatically do some simple tasks performed by nurses or caregivers in a hospital domain is designed, and a prototype is produced. The main purpose of performing these tasks with the mobile robot is to eliminate the confusion that may occur during drug delivery to the patients, make this process automatic and recordable, and keep the 
patient's morale at the highest level by the robot's voice interaction. Also, a controller is designed to track the trajectories that will provide navigation to the patient rooms on the developed scenario map, and simulations are carried out with MATLAB. The position values of the trajectories tracked by the mobile robot and the control velocity values required to go these positions are taught to two different artificial neural networks. Thus, trajectories are taught once with line tracking, and then the robot is enabled to navigate without the need for lines. Artificial neural network studies are also revealed that the RBNN type network is more successful. In future studies, the controller design can be improved, and different control structures can be tested. Besides, mass production can be started for the mobile robot.

\section{Acknowledgements}

This research results consisted of a part of project number FOA-2013-4150. The authors would like to express their thanks to Erciyes University-Scientific Research Projects Unit (BAP) for supporting this project.

\section{References}

[1] Klancar, G., Zdesar, A., Blazic, S., \& Skrjanc, I. (2017). Wheeled Mobile Robotics: From Fundamentals Towards Autonomous Systems. Butterworth-Heinemann.

[2] Vepa, R. (2009) Biomimetic Robotics Mechanism and Control. Cambridge University Press, 343.

[3] Kosuge, K., Hayashi, T., Hirata, Y., \& Tobiyama, R. (2003). Dance Partner Robot - Ms DanceR. IEEE International Conference on Intelligent Robots and Systems, 4(October), 3459-3464.

[4] Wang, C., Liu, X., Yang, X., Hu, F., Jiang, A., \& Yang, C. (2018). Trajectory tracking of an omnidirectional wheeled mobile robot using a model predictive control strategy. Applied Sciences (Switzerland), 8(2).

[5] Liu, Y., Zhu, J. J., Williams, R. L., \& Wu, J. (2008). Omni-directional mobile robot controller based on trajectory linearization. Robotics and Autonomous Systems, 56(5), 461-479.
[6] Karras, G. C., \& Fourlas, G. K. (2020). Model Predictive Fault Tolerant Control for Omnidirectional Mobile Robots. Journal of Intelligent and Robotic Systems: Theory and Applications, 97(3-4), 635-655.

[7] Wang, D., Wei, W., Yeboah, Y., Li, Y., \& Gao, Y. (2020). A Robust Model Predictive Control Strategy for Trajectory Tracking of Omnidirectional Mobile Robots. Journal of Intelligent and Robotic Systems: Theory and Applications, 98(2), 439-453.

[8] Cooney, J. A., Xu, W. L., \& Bright, G. (2004). Visual dead-reckoning for motion control of a Mecanumwheeled mobile robot. Mechatronics, 14(6), 623637.

[9] Hashemi, E., Ghaffari Jadidi, M., \& Ghaffari Jadidi, N. (2011). Model-based PI-fuzzy control of fourwheeled omni-directional mobile robots. Robotics and Autonomous Systems, 59(11), 930942.

[10] Mishra, S., Sharma, M., \& Santhakumar, M. (2019). Behavioural Fault-tolerant-control of an Omni-directional Mobile Robot with Fourmecanum Wheels. 69(4), 353-360.

[11] Tan, H., Mao, Y., Xu, Y., Kannan, B., Griffin, W. B., \& Derose, L. (2016). An integrated robotic system for transporting surgical tools in hospitals. 10th Annual International Systems Conference, SysCon 2016 - Proceedings.

[12] Wang, C., Savkin, A. V., Clout, R., \& Nguyen, H. T. (2015). An intelligent robotic hospital bed for safe transportation of critical neurosurgery patients along crowded hospital corridors. IEEE Transactions on Neural Systems and Rehabilitation Engineering, 23(5), 744-754.

[13] Takahashi, M., Suzuki, T., Shitamoto, H., Moriguchi, T., \& Yoshida, K. (2010). Developing a mobile robot for transport applications in the hospital domain. Robotics and Autonomous Systems, 58(7), 889-899.

[14] Saekow, P., Neranon, P., \& Smithmaitrie, P. (2018). External force/velocity control for an autonomous rehabilitation robot. IOP Conference Series: Materials Science and Engineering, 297(1). 